\section{Prostatitis crónica/síndrome de dolor pélvico crónico. Un reto terapéutico}

\section{Chronic prostatitis/chronic pelvic pain syndrome. A therapeutic challenge}

\section{Sr. Editor:}

La prostatitis crónica/síndrome de dolor pélvico crónico consiste en un dolor en la región pélvica durante un mínimo de 3 meses, con cultivos estériles y recuentos significativos o insignificantes, de leucocitos en muestras específicas de la próstata (semen, secreciones prostáticas) y orina obtenida después de un masaje prostático. Según la clasificación del National Institute of Diabetes and Digestive and Kidney Diseases (NIDDK), la PC/SDPC es clasificada en la categoría III. Dicha categoría a su vez, se clasifica en un subtipo inflamatorio IIIa (se observan leucocitos en secreción prostática espontánea, orina posmasaje prostático o semen); y no inflamatorio IIIb (no se observan dichos leucocitos) ${ }^{1}$.

The National Institutes of Health Chronic Prostatitis Symptom Index (NIH-CPSI), ha establecido una escala para la evaluación inicial y el seguimiento de los pacientes diagnosticados de PC/ SDPC, con el fin de poder cuantificar la respuesta al tratamiento que reciben. Dicho cuestionario explora los tres grandes dominios de la prostatitis: el dolor (puntuación 0-21), alteraciones miccionales (puntuación 0-10) y el impacto en la calidad de vida (puntuación 0-12), siendo la puntuación total del NIH-CPSI de 0-43.

En ocasiones esta sintomatología afecta de modo sustancial su calidad de vida, similar a la de los pacientes con infarto de miocardio, enfermedad de Crohn, diabetes mellitus, etc. ${ }^{2}$.

Su prevalencia, etiología, fisiopatología y terapia óptima es aún incierta, pasando de ser considerado durante años un proceso infeccioso tratado únicamente con antibióticos, a actualmente un síndrome con una variedad de presentaciones, desafíos diagnósticos y terapéuticos. Se ha visto que en esta patología influyen factores fuera del ámbito de la urología, como factores neurológicos, inmunológicos y psicológicos, que lo convierten en un síndrome multidisciplinar.

Hasta hace poco, la terapia médica estándar consistía en anti-inflamatorios no esteroideos, fitoterápicos, alfa-bloqueantes, antibióticos e inhibidores de la 5-alfa-reductasa. Sin embargo, esta estrategia no ha sido uniformemente efectiva ${ }^{3}$.

En muchas ocasiones, el uso de terapias adjuntas tales como los cambios dietéticos, fisioterapia y biofeedback, acupuntura y tratamiento del estrés puede tener un impacto profundo sobre la sintomatología y potenciar así los beneficios de las terapias tradicionales ${ }^{2}$.

Existen estudios que han reportado que los alimentos más agravantes son los picantes, seguidos del café, cola, pimienta, te y "chili" (ají). Por tanto, la intervención alimentaria debería ser considerada una piedra angular en el tratamiento de este síndrome.

También se ha documentado que la PC/SDPC está asociada con desórdenes de la salud mental, tales como la depresión y la anorexia. Tratando de identificar alteraciones con la terapia cognitivoconductual podemos ayudar a los pacientes a hacer frente a sus síntomas. A pesar de que diferentes estudios muestran que dichos programas son factibles en este tipo de patología para el alivio sintomático y de la calidad de vida, sin embargo, son necesarios ensayos clínicos controlados, aleatorizados con amplias muestras y un período largo de seguimiento ${ }^{2}$.

La acupuntura, es una técnica que está ganando aceptación en un número de patologías crónicas, entre ellas la PC/SDPC, al demostrar una superioridad terapéutica respecto a otros tratamientos. Aunque su mecanismo de acción es desconocido, parece tener propiedades antiinflamatorias y mecanismos neuromoduladores ${ }^{2}$.

La disfunción de la musculatura del suelo pélvico está documentada en pacientes con PC/ SDPC y se asocia a menudo con dolor, hipertonía y sensibilidad de los músculos de la pelvis. El biofeedback y la reeducación del suelo pélvico han sido evaluados en varios trastornos de dolor crónico, incluyendo PC/SDPC.

La hipertonía y espasticidad de la musculatura del suelo pélvico puede dar lugar a puntos de activación, que son zonas hiperirritables que causan dolor selectivo cuando se comprimen. La ubicación más probable de dichos puntos de activación en los pacientes con PC/SDPC son los músculos puborrectal y/o pubococcígeo, que provocaban dolor local y/o referido en el pene en 93\% de los pacientes evaluados ${ }^{4}$.

La neuromodulación, basada en la estimulación eléctrica de raíces nerviosas, ha sido utilizada du- 
rante décadas en el tratamiento del dolor crónico. Las técnicas de neuromodulación usadas para el tratamiento de la PC/SDPC incluyen la estimulación sacra, del nervio tibial posterior y nervio pudendo, aunque sólo la estimulación de la raíces sacras y del nervio tibial posterior están aprobadas por la US Food and Drug Administration para el tratamiento de síntomas urinarios y ninguna de estas técnicas están reconocidas como terapias estándar del tratamiento del SDPC, aunque han mostrado resultados favorables ${ }^{5}$.

La electroestimulación nerviosa transcutánea se ha empleado para el tratamiento de pacientes con SDPC, obteniendo un resultado exitoso en los estudios publicados. De igual forma, se ha investigado el efecto de la estimulación magnética extracorpórea en pacientes con PC/SDPC, llegando a la conclusión de que puede ser una opción terapéutica en este tipo de pacientes.

La terapia de ondas de choque extracorpórea se está utilizando desde hace tiempo para el tratamiento de los cálculos urinarios. El mecanismo por el cual produce un efecto analgésico es desconocido, lo que la ha convertido en un enfoque terapéutico cada vez más popular para el tratamiento alternativo de una serie de afecciones de los tejidos blandos. Diferentes estudios han mostrado su eficacia en comparación con placebo, observando descensos significativos en las puntuaciones totales, subpuntuación del dolor y calidad de vida de la escala NIH-CPSI ${ }^{6}$.

Existen estudios que proponen la terapia térmica o hipertermia como una opción para el tratamiento de la PC/SDPC. Sin embargo, existen vacíos en lo que respecta al tipo de calor a utilizar (intersticial o microondas), la vía de administración (transrectal o transuretral), duración del tratamiento, etc.

En conclusión, el tratamiento de la PC/SDPC ha evolucionado rápidamente en los últimos $5 \mathrm{a}$ 10 años. Diferentes estudios han puesto de manifiesto que la PC/SDPC es un problema médico real. La alteración de la calidad de vida, asociada al coste sanitario y socioeconómico que genera, han desatado una oleada de búsqueda de una nueva generación de alternativas terapéuticas que ayuden a combatir este problema.

Antonio Jiménez-Pacheco ${ }^{1}$, PhD., Araceli Jiménez-Pacheco ${ }^{2}$ ${ }^{1}$ Servicio de Urología. Hospital Santa Ana, Granada. España.

${ }^{2}$ Servicio de Rehabilitación. Centro Traumatológico y de Rehabilitación. Hospital Universitario Virgen de la Nieves, Granada. España.

\section{Referencias}

1. Litwin MS, McNaughton-Collins M, Fowler FJ Jr, Nickel JC, Calhoun EA, Pontari MA, et al. The National Institutes of Health chronic prostatitis symptom index: development and validation of a new outcome measure. Chronic Prostatitis Collaborative Research Network. J Urol 1999; 162 (2): 369-75.

2. Amin S. Herati, Robert Miles Moldwin. Alternative therapies in the management of chronic prostatitis/chronic pelvic pain syndrome. World J Urol 2013; 31 (4): 761-6.

3. Schaeffer AJ. Clinical practice. Chronic prostatitis and the chronic pelvic pain syndrome. N Engl J Med 2006; 355 (16): 1690-169.

4. Anderson RU, Sawyer T, Wise D, Morey A, Nathanson BH. Painful myofascial trigger points and pain sites in men with chronic prostatitis/chronic pelvic pain syndrome. J Urol 2009; 182 (6): 2753-8.

5. Yang CC. Neuromodulation in male chronic pelvic pain syndrome: rationale and practice. World J Urol 2013; 31 (4): 767-72.

6. Xiao-yong Z, Chen L, Zhang-qun YE. Extracorporeal shock wave treatment for non-inflammatory chronic pelvic pain syndrome: a prospective, randomized and sham-controlled study. Chin Med J 2012; 125 (1): 114-8.

Los autores declararon no tener conflictos de intereses.

Correspondencia a:

Antonio Jiménez-Pacheco

Servicio de Urología. Hospital Santa Ana. Avenida Enrique Martín Cuevas s/n. Motril. Granada, España. CP: 18600.

Teléfono: 958038420

Dirección postal: Calle Párroco José Rodríguez 65, Bloque A1

3B. Granada. CP: 18014. Teléfono: 958154355/615100625

anjipa29@hotmail.com 